\title{
La nouvelle architecture de l'industrie de la Défense en France
}

Évolution du rôle du maître d'ouvrage

Nathalie Lazaric, Valérie Mérindol et Sylvie Rochhia

\section{(2) OpenEdition}

\section{Journals}

Édition électronique

URL : http://journals.openedition.org/ei/267

DOI : $10.4000 /$ ei. 267

ISSN : 2553-1891

Éditeur

Association Économie et Institutions

Édition imprimée

Date de publication : 30 octobre 2009

Pagination : 31-60

ISSN : 1775-2329

Référence électronique

Nathalie Lazaric, Valérie Mérindol et Sylvie Rochhia, « La nouvelle architecture de l'industrie de la Défense en France », Économie et institutions [En ligne], 12-13 | 2009, mis en ligne le 31 janvier 2013 consulté le 19 avril 2019. URL : http://journals.openedition.org/ei/267 ; DOI : 10.4000/ei.267 


\title{
La nouvelle architecture de l'industrie de la Défense en France: Evolution du rôle du maître d'ouvrage
}

\author{
Nathalie LAZARIC ${ }^{1}$, Valérie MERINDOL 2 \\ et Sylvie ROCHHIA ${ }^{3}$
}

\section{INTRODUCTION}

La complexité technologique, l'introduction massive des Technologies de l'Information et de la Communication (TIC) dans les systèmes d'armes sont emblématiques des changements apparus au sein de l'industrie de la Défense. En accentuant le poids des réformes en cours, en obligeant à clarifier le rôle de l'Etat et des firmes dans la conception des programmes, les variables technologiques ont accentué l'émergence de nouveaux modes de production et de coordination des actifs.

Parce que la connaissance et la compétence sont de plus en plus distribuées, la conception et l'élaboration d'un programme d'armement nécessitent une coopération et une coordination entre les acteurs publics et privés. Cette division du travail repose sur la nécessité de combiner des compétences proches et/ou complémentaires et génère ce que Simon (1991) appelle une hiérarchie arborescente, ou architecture, dont la "décomposabilité " est une propriété essentielle. Le concept d'architecture industrielle qui désigne la division du travail entre firmes et les institutions, ainsi que les mécanismes d'allocation de ressources et de coordination entre ces différentes organisations (Jacobides \& al. 2006 ; Jacobides, 2006), permet de rendre compte de ce processus.

Depuis le débat lancé par Simon sur la décomposition des systèmes et leur modularité, le sens de la causalité entre division du travail et division des connaissances est devenu une question centrale (Baldwin \& Clark, 2000; Brusoni \& Prencipe, 2006). Les changements structurels ayant affecté l'industrie de la Défense en France au cours des années 1990 (Serfati, 2001, 2008) illustrent bien cette question. En effet, la division du travail et des connaissances a été modifiée mais les causes de ces changements restent encore mal identifiées. $\mathrm{Si}$ les facteurs technologiques s'avèrent essentiels pour comprendre l'évolution de cette architecture, les facteurs institutionnels ont aussi joué un rôle

1 GREDEG, UMR 6227 CNRS, Université de Nice Sophia Antipolis

2 CERNA, Ecoles des Mines ParisTech, et IMRI, Université Paris IX Dauphine

3 GREDEG, UMR 6227 CNRS, Université de Nice Sophia Antipolis

31 Economie et Institutions - $\mathrm{n}^{\circ} 12 \& 13-2008-2009$ 
essentiel. La conception et la production d'armement nécessitent, en effet, l'émergence de règles qui favorisent le développement de relations durables ainsi que le transfert de connaissances. Le recours à la contractualisation est loin d'être simple car beaucoup des soussystèmes ou actifs concernés s'avèrent co-spécifiques (Teece 1986 ; O’Sullivan 2006), d'une grande variété et complexité.

Dans la plupart des pays, l'architecture de l'industrie de la Défense se structure autour de deux acteurs essentiels: les firmes intégrateurs de systèmes et l'Etat, client et maître d'ouvrage des programmes. Ce dernier occupe un rôle important pour la coordination des actifs co-spécifiques. En France, la responsabilité de la maîtrise d'ouvrage des programmes d'armement est traditionnellement confiée à la Délégation Générale pour l'Armement (DGA). Cette institution a notamment permis à partir des années 1960, l'émergence d'une industrie de haute technologie capable de concevoir tous les grands programmes militaires français. Au cours des années 1990-2000, d'importants changements technologiques et institutionnels ont introduit une évolution fondamentale des relations entre les firmes et la DGA.

Dans cet article, nous montrerons comment la co-évolution de ces éléments technologiques et institutionnels a été à l'origine d'une nouvelle architecture de l'industrie et un repositionnement de la DGA. Cette recherche repose sur la réalisation de plus d'une quarantaine d'entretiens qualitatifs réalisés auprès de responsables travaillant au sein de la DGA, des entreprises en charge de la conception des programmes ainsi que des centres de R\&D travaillant pour la Défense entre 2000 et $2008^{4}$ (cf annexe 2).

Dans la première partie, nous mettrons l'accent sur les éléments d'ordre technologique ayant contribué à définir la nouvelle architecture de cette industrie. Dans la seconde partie, nous mettrons en évidence l'incidence des reformes sur les comportements et rôles des acteurs publics et privés, et plus particulièrement sur la situation de la DGA. Dans la troisième partie, nous montrerons comment le rôle de maître d'ouvrage de la DGA s'est modifié. La remise en cause des connaissances et des compétences détenues par cette institution ayant été notable, nous conclurons sur les conséquences de cette évolution pour l'ensemble de l'industrie.

4 Une partie de ces entretiens fut réalisée et financée par deux études de l'OED (Observatoire Economique de la Défense) : "Innovation, diffusion des connaissances et croissance: le cas des entreprises du secteur de la défense", DAF/OED/2001/ et "Typologie des compétences des entreprises liées à la défense : analyse des compétences codifiées et des compétences non codifiées". Une autre partie des entretiens a été conduite au sein de l'Observatoire Economique de la Défense (OED) et du Centre de Recherche de l'Armée de l'Air dans le cadre d'études internes.

32 Economie et Institutions - n²12\&13-2008-2009 


\section{ARCHITECTURE DE L'INDUSTRIE DE LA DEFENSE ET CHANGEMENT TECHNOLOGIQUE}

L'architecture de l'industrie de la Défense se caractérise par une division hiérarchique du travail entre l'Etat client, la firme intégrateur de systèmes et les sous-traitants, ainsi que par des programmes dont la plupart sont aujourd'hui qualifiés de complexes (Davies \& Hobday, 2005).

\subsection{Architecture et systèmes complexes (CoPS5)}

La complexité d'un produit peut être appréhendée à travers plusieurs dimensions telles que le nombre de composants, la diversité des inputs matériels et informationnels, le degré de "surmesure " à la fois du système et des sous-systèmes ainsi que la complexité de l'architecture du système ${ }^{6}$ (Hobday, 2000). La nature des interfaces permet de caractériser la complexité de l'architecture système. Dans le cas des systèmes d'armes, les sous-ensembles interagissent et sont, le plus souvent, interdépendants ; l'architecture tend alors à être intégrale ${ }^{7}$ (Ulrich, 1995).

Les systèmes sont, en effet, organisés en sous-systèmes confiés à un nombre restreint de sous-traitants directs, à charge pour ces derniers d'organiser leur propre sous-traitance en cascade. En France, cette division du travail a pris corps progressivement dès les années 1960 avec la conception du Mirage IV et l'émergence du statut d'intégrateur de système pour la firme responsable du programme (Versailles, 2005). Pendant plusieurs décennies, l'architecture de l'industrie va être marquée par une spécialisation des actifs assez prononcée au sein des réseaux (Avadykian et al., 2005).

En général, l'Etat occupe la fonction de maître d'ouvrage des programmes ce qui renvoie au rôle de l'acteur public dans le choix de l'architecture technologique de ces grands programmes ${ }^{8}$ (Mérindol 2005a ; Belleval 2006). Plus précisément, la responsabilité centrale

5 Complex Products and Systems.

6 L'architecture d'un système décrit les différents sous-systèmes ou modules qui composent un système ainsi que les relations entre ces entités. Et comme l'a montré Simon (1991), la création d'une telle architecture passe par un processus de décomposition des fonctions en sous fonctions.

7 Selon Ulrich (1995), lorsqu'un système complexe peut être décomposé en sous-systèmes autonomes (modules), ces derniers peuvent être reliés par des interfaces couplées (architecture intégrale) ou découplées (architecture modulaire).

8 De façon classique, cela signifie que l'Etat détermine le cahier final des charges en spécifiant le besoin, les objectifs et les contraintes associées au projet.

33 Economie et Institutions - n² 12\&13-2008-2009 
de la maîtrise d'ouvrage des programmes d'armement français est assurée par la $\mathrm{DGA}^{9}$ qui doit être capable d'arbitrer entre différentes options scientifiques et technologiques tout en prenant en compte l'évolution des coûts et la satisfaction des besoins militaires. Cette institution joue un rôle clé dans la définition et le choix des propriétés attendues des systèmes fournis aux Armées puisque c'est elle qui, en amont, définit les critères de choix et de contrôle du travail confié à l'entreprise maître d'œuvre et intégrateur de systèmes.

Pour comprendre ces différentes fonctions, il peut être utile de se référer à la typologie Architectural knowledge/ Component knowledge proposée par Hobday (1998). Les connaissances composants (Component knowledge) renvoient aux savoirs spécifiquement associés aux briques technologiques réalisées par les sous-traitants et, qui sont intégrées dans tout système. La fonction d'intégration de système repose sur des connaissances architecturales (Architectural knowledge). Ces connaissances permettent de savoir combiner et intégrer une variété très grande de technologies lors de la conception d'un système complexe. Les connaissances architecturales sont indissociables du métier d'intégrateur qui nécessite des compétences technologiques et organisationnelles préalables pour être à même de piloter le système (Prencipe, 1997; Davies \& Brady, 2000; Hobday, 2000; Hobday et al. 2005). Grâce à sa connaissance des champs technologiques sousjacents aux composants ou sous-systèmes confiés en sous-traitance, l'intégrateur est capable de concevoir et tester le système mais aussi de faire face aux interactions imprévues entre les composants ainsi qu'à la diversité des rythmes de développement des composants et sous-systèmes.

L'étude de Prencipe (2000) sur l'industrie aéronautique montre que toute décision majeure concernant l'Architectural knowledge nécessite de maîtriser des connaissances au niveau du Component knowledge. L'adage selon lequel "firms know more than they make" (Brusoni et al. 2001) prend alors tout son sens dans l'industrie de la Défense. L'intégrateur doit, en effet, comprendre les incidences possibles de l'architecture du système sur le développement des différentes briques technologiques qui composent le programme. Dans le cas d'une architecture système intégrale, cette exigence est essentielle. Ainsi, l'intégrateur ne se contente pas de proposer une structure globale de conception des programmes complexes, il est aussi capable de choisir et de faire évoluer les différentes options technologiques qui se présentent. Les

9 La DGA comprend aujourd'hui environ 19000 personnes (6500 dans des postes de direction et de manageurs, 9600 dans des structures de tests, d'essais et d'expertises et 3400 dans des activités de maintenance).

34 Economie et Institutions $-\mathrm{n}^{\circ} 12 \& 13-2008-2009$ 
connaissances et compétences nécessaires à la fonction d'intégration se définissent donc comme :

'.the capabilities which enable firms, government agencies, regulators, and a range of other actors to define and combine together all the necessary inputs for a system and agree on a path of future systems development. In the narrower sense of firm capability, system integration is concerned with the way in which firms and others agents bring together hightechnology components, subsystems, software, skills knowledge engineers, managers, and technicians to produce a product in competition with other supplier. (...) systems integration capability is not merely the counterpart to outsourcing, but the capability needed to manage outsourcing as well as 'joint sourcing' and 'insoursing' to enable the systems integrator firm to gain the advantages of both outsourcing and vertical integration through different phases of the product life cycle' (Hobday et al., 2005: 1110-1111).

Les connaissances architecturales peuvent aussi être maîtrisées par le maître d'ouvrage. Celui-ci est amené à travailler avec la firme intégrateur de système dans une logique de cospécification, par exemple pour les interfaces entre sous-systèmes interdépendants. L'architecture de l'industrie de la Défense s'est donc construite sur la base d'une intégration de systèmes partagée entre la firme intégrateur de systèmes et l'Etat maître d'ouvrage (Hobday et al., ibid). Cette partition est importante car l'architecture ne reflète pas seulement le processus de division du travail entre les différents acteurs, elle reflète également le processus de définition et d'appropriation de la valeur au sein de l'industrie (Jacobides et al., 2006). Au-delà des modalités de production, l'architecture d'une industrie renvoie aussi aux problèmes de contrat et d'incitation.

Avec la complexité technologique croissante des produits, cette coordination peut évoluer. La complexité reste une question de degré, mais il s'avère que plus les systèmes sont complexes, moins ils peuvent s'accommoder d'une mise en concurrence classique. En effet, tout changement de "design" ou de sous-traitants risque d'entraîner d'importantes altérations des autres modules, nécessitant la mise en place de systèmes de contrôle plus sophistiqués. En d'autres termes, plus la complexité des systèmes s'accroît, plus la contrepartie en terme de coordination est forte. Or, au cours des années 1990, la complexité dans la conception des programmes d'armement se renforce avec le développement des "systèmes de systèmes". 


\subsection{De la complexité aux " systèmes de systèmes "}

Les "systèmes des systèmes " peuvent être définis comme des ensembles de systèmes interconnectés par des systèmes d'information et de communication (Matthew \& Collier, 2000). Dans ce cadre, il ne s'agit pas tant d'améliorer chacun des systèmes mais de maîtriser les connexions entre ces derniers ${ }^{10}$. Ces exigences nouvelles se conjuguent à une stabilité moins importante des architectures et au maintien de composants peu standardisés. L'incertitude sur la conception des programmes devient donc croissante et la maîtrise des systèmes d'information de plus en plus critique dans la gestion des programmes d'armement. Le programme français de Système de Communication et de Commandement des Opérations Aériennes (SCCOA) en constitue une illustration majeure ${ }^{11}$.

Que ce soit aux Etats-Unis puis en Europe, le développement des "systèmes de systèmes " apparaît concomitant avec l'émergence d'industriels occupant la position de "Lead Systems Integrator " (désigné après LSI) (Bailey \& Grasso, 2007). Ce dernier désigne l'industriel qui est responsable de la définition de l'architecture technologique du programme et, qui assure les fonctions de contrôle et d'évaluation du projet. Le rôle conféré au LSI offre d'une part, l'opportunité de tirer partie plus rapidement de la valeur créée, mais risque, d'autre part, d'amoindrir la position traditionnelle du maitre d'ouvrage. Dès lors, la fonction de la DGA, pourrait évoluer vers une simple gestion des interfaces, avec le risque de ne plus disposer des garanties nécessaires pour accéder aux informations techniques (ou à celles concernant le choix des entreprises sous-traitantes par le LSI).

Avec les "systèmes de systèmes ", les asymétries informationnelles tendent en effet à se renforcer entre firme LSI et maître d'ouvrage, créant de potentielles tensions dans la coordination du travail. Dans un tel contexte, la maîtrise du cadre contractuel devient un élément central dans la gestion des

10 L'étude des interdépendances entre les différents systèmes d'armes montre que les exigences opérationnelles des différentes plateformes sont étroitement définies les unes par rapport aux autres, renforçant ainsi l'importance des contraintes d'interopérabilité entre les différents soussystèmes.

11 Ce programme de 1997, qui intègre de nouveaux logiciels et systèmes de liaisons de données, est un système de systèmes devant permettre de relier l'ensemble des armements de l'Armée de l'air. La DGA a complètement délégué la conception de l'architecture technologique de ce programme à un consortium industriel formé par Aérospatiale Matra (par la suite EADS) et Thomson CSF (devenu par la suite Thales) qui occupent une fonction conjointe de Lead System Integrator (LSI).

36 Economie et Institutions - $\mathrm{n}^{\circ} 12 \& 13-2008-2009$ 
programmes. Le rôle du maître d'ouvrage tend à se limiter à la définition des attentes technico-opérationnelles et à la définition du cadre contractuel vis-à-vis des Etats majors (Flood \& Richard, 2006). Autrement dit, il finit par se comporter comme un client qui tente de peser sur l'architecture industrielle par des règles d'attribution des contrats et par la mise en place de consortia pour infléchir la coopération ou la concurrence entre les firmes (Depeyre \& Dumez, 2007).

Avec l'émergence des "systèmes de systèmes ", le métier d'intégrateur nécessite des compétences technologiques et organisationnelles qui représentent une capacité d'absorption minimale pour être à même de piloter le système (Prencipe, 1997; Davies \& Brady, 2000; Hobday, 2000; Hobday et al. 2005). Pour les LSI, l'ingénierie système ne peut plus se limiter à l'intégration matérielle du système; la dimension organisationnelle devient de plus en plus centrale ce qui engendre une évolution des connaissances et des compétences.

Ces nouvelles compétences technologiques et organisationnelles deviennent en effet essentielles pour contrôler et intégrer le travail collectif entre un nombre croissant de partenaires. La division du travail étant rarement identique à celle des connaissances, l'influence de la firme intégrateur de systèmes dans la gestion des spécifications techniques du projet et des contrats industriels se révèle déterminante. A titre d'exemple, la mise en œuvre des plateformes technologiques communes ${ }^{12}$ peut faciliter les interactions et la potentielle combinaison des actifs développés lors de ces projets ou des programmes. En effet, la spécialisation croissante des compétences nécessite diverses formes d'alliances pour réaliser les tâches qui auparavant pouvaient se faire sous la maîtrise d'une seule firme. Conjuguée à la complexité technologique, cette spécialisation pousse donc les firmes à combiner les deux modalités suivantes :

(1) Continuer à faire faire certaines tâches pour ensuite les intégrer au sein de l'ensemble technologique à réaliser. Cette première option renvoie à la logique classique de division du travail qui a caractérisé l'architecture industrielle jusqu'à la fin des années 90 . La conception d'un système d'armes tendait alors à se réduire à la définition

\footnotetext{
12 Ces plateformes permettent par exemple à une firme spécialisée dans les matériaux de réaliser une solution spécifique pour EADS, intégrateur de systèmes aéronautiques et spatiaux. Cette solution technique sera exécutée dans la grande firme aéronautique et pourra être redéployée ailleurs si nécessaire.
}

37 Economie et Institutions - n²12\&13-2008-2009 
d'une architecture, puis à la sous-traitance de la conception et production des sous systèmes et composants.

(2) Avoir recours à des actifs co-spécialisés, c'est-à-dire cospécifiés ensemble et exécutés ensemble selon la complémentarité des ressources technologiques ou organisationnelles. Cette seconde option se renforce avec l'émergence des "systèmes de systèmes " et devient essentielle pour comprendre les enjeux de la nouvelle architecture.

La complexification croissante suppose une combinaison des actifs variant en fonction des modalités de résolution des problèmes identifiés par les acteurs. Le contenu des actifs peut modifier la nature des interactions et les modèles d'innovation (cf. à cet égard Chesbrough, 2003). Du point de vue des stratégies d'acteurs, la nouvelle architecture de l'industrie permet donc de se repositionner sur la chaîne de valeur. Dans ce contexte, la création d'actifs cospécialisés, souvent nécessaire pour bénéficier de ressources complémentaires, devient centrale (Teece, 2006). Définis comme " those with bilateral dependence" (Teece, 1986 : 289), ces actifs sont ceux dont la combinaison offre des externalités positives liées à la complémentarité des produits, services ou processus. Dans l'approche traditionnelle (Teece, 1986), ces actifs sont relativement immobiles car la co-spécialisation suppose une forte complémentarité et une faible mobilité entre les entreprises. Pourtant, rien n'empêche leur déplacement afin de mieux tirer partie de leur complémentarité et de leur exploitation (Jacobides et al., 2007). L'objectif étant d'exploiter la meilleure combinaison des ressources existantes et de minimiser les coûts de transaction. Ainsi,

"disentangling the two constituent components of cospecialization gives rise to the new insight that firms manage to obtain both high complementarity and high mobility in their vertically adjacent segments can appropriate value without owning the complementary asset, thus evading the canonical Teecian co-specialization conundrum" (Jacobides et al., 2006:1201).

Ainsi, il existe diverses modalités pour créer des actifs cospécialisés et bénéficier des mutations de l'architecture de l'industrie de la Défense. Les changements opérés au sein du système national d'innovation (après SNI) induisent le repositionnement des institutions, redessinent de nouvelles interactions entre ces dernières et offrent de nouvelles opportunités dans la création d'actifs qui vont être étudiés dans la section suivante. 


\section{ARCHITECTURE DE L'INDUSTRIE ET CHANGEMENT INSTITUTIONNEL}

L'efficacité technologique des organisations dépend aussi du fait qu'elles soient isomorphes avec leur environnement institutionnel (Dimaggio \& Powell, 1983). Les sources du changement institutionnel sont multiples. Elles peuvent être exogènes, résultant d'une évolution du contexte technologique et politique. C'est le cas de la réforme de la politique d'acquisition d'armement en 1997, contrecoup de la prise en compte de nouvelles contraintes budgétaires et de la complexité technologique croissante. Les sources du changement peuvent être endogènes, c'est-à-dire être la conséquence du comportement des acteurs lorsque le contexte institutionnel n'est pas aligné avec leur intérêt ou devient incompatible avec l'évolution économique et technologique. Seo et Ceed (2002) mettent en perspective l'existence d'un découplage potentiel entre la légitimité institutionnelle et l'efficacité d'un point de vue technologique. Les règles institutionnelles ne sont plus alors perçues comme cohérentes avec la survie de l'organisation. La réforme de 2003 de la DGA procède d'une telle approche. Elle modifie les impacts de la réforme de 1997.

\subsection{La réforme de 1997 : la fin du recours au marché administré}

Jusqu'au milieu des années 1990, l'armement représentait un marché administré caractérisé par le recours quasi systématique aux contrats de type "cost plus " (Chesnais \& Serfati, 1992; Hartley, 1995). La mise en concurrence des firmes, lorsqu'elle était possible, restait à la discrétion de l'administration et restreinte aux seules entreprises nationales ${ }^{13}$. Dans la plupart des cas, les contrats comportaient des obligations de sous-traitance en faveur de la firme non retenue créant ainsi des incitations aux stratégies d'alliances.

De nouvelles règles en matière d'acquisition d'armement

La réforme de 1997 fut le résultat de la prise en compte du nouveau contexte géostratégique et budgétaire de l'après Guerre froide. Ainsi, le budget de Recherche et Développement militaire (R\&D) est passé de 4850 millions d'euros (constants 2004) en 1996, à 3636 millions d'euros en 2004. Pour la part consacrée à la R\&T14,

13 Selon Kirat, en 1994, la procédure négociée était utilisée pour $94 \%$ du montant total des commandes et $80 \%$ de ces marchés étaient passés sans mise en concurrence (Kirat et al., 2003).

14 Le budget de Recherche et Technologie (R\&T) constitue un agrégat retraité au sein du ministère de la Défense qui permet de se rapprocher de la définition de la R\&D au sens du manuel de Frascati de l'OCDE. Il comprend

39 Economie et Institutions - n²12\&13-2008-2009 
le budget est passé de 650 millions d'euros en 1996 (constant 2004) à 380 millions d'euros en 2004 (constant 2004) soit une diminution de près de $50 \%$ en moins de 10 ans. Cette réforme fut aussi la conséquence d'une évolution majeure du SNI (Larédo \& Mustar, 2001) car elle remet en cause les politiques de grands programmes technologiques et conduit à de nouvelles priorités en matière de politique publique. Les changements se traduisent non seulement par la privatisation firmes liées à la Défense mais aussi par l'introduction de nouvelles règles du jeu entre la DGA et les firmes (Guillou et al. 2009).

A partir de cette réforme, les critères d'attribution des contrats de conception de programmes sont fondés sur la recherche du meilleur rapport coût-qualité. La réalisation des programmes est confiée aux entreprises sur la base de contrat à prix fixe, transférant ainsi la totalité des risques financiers et technologiques au secteur privé. Cette évolution de la politique d'acquisition s'inspire de la réforme "Levine ", instaurée par le ministère de la Défense britannique quelques années auparavant, lors de l'introduction de la politique dite du "best value for money". En France, elle mit fin à une politique d'autonomie complète dans la production d'armement, qui fut le fondement de la politique de la DGA depuis sa création en 1961. Le principe d'une concurrence ouverte aux entreprises étrangères, principalement européennes implique l'instauration de nouvelles relations de "marché " entre le ministère de la Défense et les entreprises (DGA, 1997). L'intensité des échanges informels se réduit, conséquence de la formalisation des procédures de mise en concurrence des marchés publics (Kirat et al., 2003).

Les incidences sur la division du travail

Cette réforme a aussi accéléré la réorganisation des actifs car les groupes industriels voient leur rôle se renforcer dans l'exécution de la recherche et dans la coordination des tâches. Les firmes se repositionnent donc sur la chaîne de valeur à travers un double mouvement d'intégration et de désintégration verticale pour mieux tirer profit de leurs innovations (Dowdall, 2004 ; Acha \& Brusoni, 2008 ; Hobday et al., 2005 ; Guillou et al., 2009) et des opportunités de ces changements. Alors que le rôle des firmes s'affirme dans la réalisation des programmes d'études amont, celui de la DGA se recentre sur le suivi de la conception des programmes complexes et sur des activités plus aval, liées aux tests et essais des programmes en phase d'opérationnalisation (Guichard, 2005). Sauf en ce qui

le budget consacré aux travaux de recherche fondamentale et appliquée. Généralement l'agrégat R\&D militaire intègre des crédits de développement industriel qui ne sont pas intégrés dans les budgets civils de R\&D.

40 Economie et Institutions $-\mathrm{n}^{\circ} 12 \& 13-2008-2009$ 
concerne le domaine nucléaire, ce sont donc les groupes industriels qui vont assurer l'interface avec les réseaux de recherche scientifiques et technologiques (Mérindol, 2005b).

\title{
Le positionnement de la DGA comme centrale d'achat
}

Avant 1997, la DGA avait en charge la préservation des compétences industrielles sur le long terme ce qui lui conférait le monopole des interfaces entre le ministère de la Défense, l'industrie et les réseaux scientifiques pour l'élaboration des programmes d'armement. La maîtrise d'ouvrage reposait alors sur un arrangement institutionnel complexe entre les différentes organisations dépendantes du Ministère de la Défense ou étant sous sa tutelle ${ }^{15}$. A partir de 1997 , le modèle de référence pour la DGA devient celui de la centrale d'achat plutôt que celui de maître d'ouvrage ${ }^{16 .}$.

Une distinction claire est donc opérée entre la politique technologique (services centraux de la politique industrielle) et la politique d'achat (direction de programmes) (Giovachini, 2000). Les considérations de politiques technologiques passent au second plan par rapport aux besoins opérationnels sur la base d'un meilleur rapport coût/qualité (Kausal et al., 2001). La réforme de 1997 est présentée dans le graphique 1.

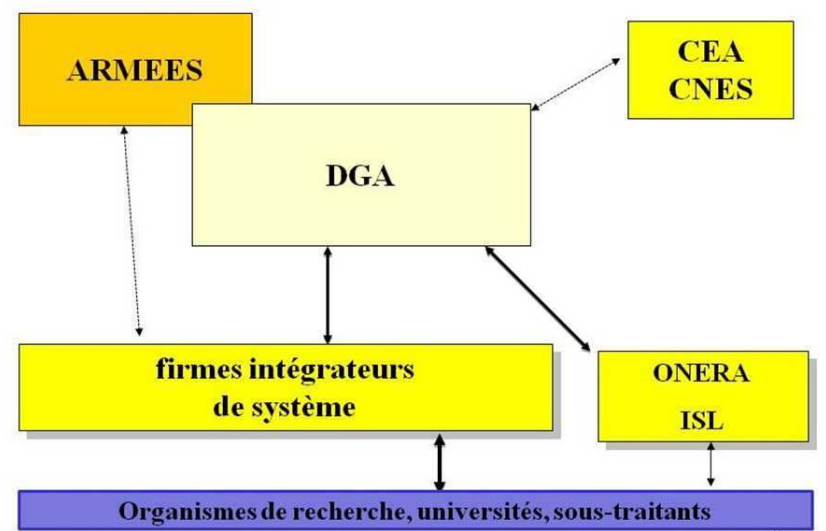

Figure 1 - La situation de la DGA après la réforme de 1997

\begin{abstract}
15 Des expertises variées étaient donc mobilisées au sein des Armées, de la Direction des applications militaires du CEA, de l'ONERA et du CNES.

${ }^{16}$ Les discours de J.Y Helmer, Délégué général de la DGA, en charge de la mise en œuvre de la réforme sont à ce titre éloquents. La référence au terme de maitre d'ouvrage est totalement bannie des discours officiels. La référence principale est associée au modèle de la centrale d'achat de Peugeot, réforme conduite par J.Y Helmer lui-même avant de prendre le poste de Délégué Général de l'Armement
\end{abstract}

41 Economie et Institutions - n² 12\&13-2008-2009 


\subsection{La réforme de 2003-2004 : relations partenariales et réaffirmation de la fonction de maîtrise d'ouvrage}

Le constat d'une perte importante de compétences industrielles et technologiques (Linière Cassou, 2000) va conduire à une nouvelle évolution de la politique d'acquisition et de R\&D militaire. En effet, au début des années 2000, la DGA devient de plus en plus dépendante de la recherche exécutée par les firmes ce qui pose la question du maintien de son expertise.

\section{La réaffirmation du statut de maître d'ouvrage pour la DGA}

Le premier volet de la réforme va consister à revoir l'implication de la DGA dans le développement des programmes d'armements. La fonction de maître d'ouvrage est à nouveau valorisée pour renforcer la responsabilité de la DGA dans la maitrise des risques financiers et technologiques. La garantie de la pérennité des compétences technologiques nécessaires aux futurs programmes est également affirmée comme une priorité de politique publique.

Une des implications directes de cette réforme concerne l'augmentation des crédits amont. Si cette augmentation a surtout bénéficié aux grandes firmes, la DGA a relancé des projets exploratoires avec le CNRS, l'Université et les PME. Dans sa mission pour la recherche et l'innovation scientifique, la DGA pilote annuellement 12 millions d'euros pour des projets exploratoires. Si les montants sont limités, ces initiatives traduisent une nouvelle orientation de la politique de R\&D militaire. Pour la DGA, le renforcement du suivi du contenu des travaux de conception est considéré comme une nécessité. Ce suivi des recherches amont permet de mieux adapter les recherches exécutées par le privé aux besoins militaires et augmente, par ce biais, la capacité d'innovation du système d'armement. Progressivement, la DGA retrouve un rôle dans la gestion des projets à vocation duale, non seulement dans les montants consacrés mais aussi dans leur pilotage. Elle participe aux projets financés par l'Agence Nationale de la Recherche, aux financements des RRIT, et aux pôles de compétitivité (cf. Serfati, 2008). Le graphique 2 ci après résume cette tendance. 


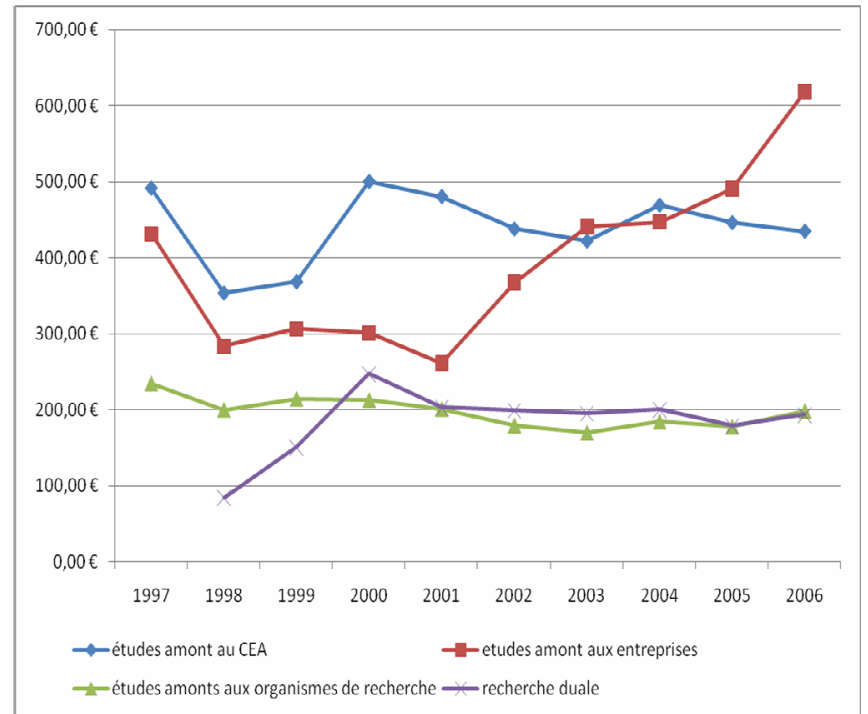

Figure 2 - Dépenses publiques de R\&T de Défense, par destinataire (ME constants 2004)

Sources : DGA et Observatoire économique de la Défense et traitement des auteurs

\section{Les difficultés rencontrées}

Cette réforme, qui a ainsi entraîné une redéfinition du périmètre des activités de la DGA et une réaffirmation de sa fonction de maître d'ouvrage, engendre pourtant des difficultés (cf. Bernard \& Carré, 2005). En effet, le pilotage des projets amont implique le renforcement de ses compétences techniques ${ }^{17}$ et conduit la DGA à avoir recours à de nouvelles modalités pour absorber les connaissances. Via des conventions d'assistance à la maîtrise d'ouvrage, elle mobilise de nouvelles activités dans les centres de recherche dont elle a la tutelle. Il s'agit de l'ONERA spécialisée dans la recherche aéronautique et la Direction des Applications Militaires (DAM) du CEA ${ }^{18}$. Le rôle attribué à l'ONERA dans les démonstrateurs de Drones miniatures obéit à cette logique (Fromion, 2005 : 46). La

17 L'un des mandats de la nomination de François Lureau comme délégué général pour l'armement en 2003 porte explicitement sur le renforcement des compétences techniques de cette institution. Cette évolution est parfois considérée comme une condition de survie de l'institution.

18 Une nouvelle convention a été signée entre la DGA et la Direction des applications militaires (DAM) du CEA. Elle permet de mobiliser les expertises du $C E A / D A M$ en élargissant leurs missions et prérogatives aux domaines ne concernant pas seulement nucléaire. La DAM a alors un rôle d'interfaces et d'orientations stratégiques des travaux confiés aux universités et aux centres publics de R\&D dans des domaines scientifiques et technologiques clés pour la Défense.

43 Economie et Institutions $-\mathrm{n}^{\circ} 12 \& 13-2008-2009$ 
convention développée entre la DGA et le CEA ou encore avec l'ONERA, correspond finalement à un modèle de capacité d'absorption externalisée définie par Cohen et Levinthal (1990). La DGA fixe l'objectif général, le budget et l'affectation des grandes masses mais confie l'ensemble de la conception des projets et l'animation des réseaux d'innovation à l'ONERA ou au CEA/DAM. Toutefois, cette capacité d'absorption externalisée peut introduire une difficulté croissante pour s'adapter aux besoins militaires dans les projets exploratoires ${ }^{19}$. En effet, la traduction des spécificités militaires en programmes technologiques est rendue difficile par la multiplication des interactions entre les centres de recherches publics et l'absence de connaissances suffisamment précises sur les besoins militaires.

L'introduction de nouvelles relations partenariales entre la DGA, les Armées et l'industrie

Confrontée à l'émergence de nouveaux domaines technologiques ${ }^{20}$ pour lesquels la DGA n'a pas su développer une variété de relations (Mérindol, 2005b), sa capacité de coordination se trouve également réduite. C'est pourquoi avec la réforme de 2003, la DGA est incitée à animer de nouveaux réseaux. Cette réforme vise à l'introduction d'une plus grande variété de relations entre les acteurs publics et privés (cf. le graphique 3). Ainsi, les rencontres Sciences et Défense, l'institutionnalisation de groupes de travail avec les agences comme le CEA, le CNES, le CNRS et l'INRIA (DGA, 20042006) montrent la volonté d'impliquer davantage d'acteurs dans les projets de R\&D militaire.

A partir de la réforme de 2003, une relation partenariale entre la DGA, les Armées et l'industrie a aussi été introduite impliquant une évolution de la gouvernance des programmes technologiques. Un pilotage direct par l'Etat Major des Armées a été mis en place pour les décisions associées aux arbitrages entre les programmes d'armement concernant le choix et les orientations budgétaires et technologiques.

La relation partenariale est essentiellement orientée en faveur des grands intégrateurs de systèmes, l'objectif étant de mieux gérer la complexité et l'incertitude associées à la conception des programmes d'armement ${ }^{21}$. La création des laboratoires technico-

19 Eléments apparus lors de la réalisation de plusieurs entretiens réalisés auprès de responsables de l'ONERA, du CEA, de l'industrie et des Armées.

20 Logiciels, Biotechnologies, Nanotechnologies

21 Ces relations ont pris la forme de rencontres institutionnalisées instituées au plus haut niveau des élites politiques et économiques. Le contenu des échanges y est assez formel. Une relation plus "libre et ouverte " est progressivement initiée autour des projets d'études amont entre la DGA et

44 Economie et Institutions - n²12\&13-2008-2009 
opérationnels (LTO) favorisant l'émergence de nouvelles relations collaboratives entre la DGA, les Armées et les firmes pour expérimenter et évaluer la technologie dans les situations de combat, en est une bonne illustration. En effet, les LTO constituent de nouvelles plateformes collaboratives fondées sur des modèles hybrides de gouvernance de la R\&D avec des mécanismes de marché et des relations de confiance. Ils se sont constitués, d'une part pour l'expérimentation et la simulation des technologies dans le cadre des exercices opérationnels, et d'autre part, autour d'une dynamique nouvelle de co-conception des projets sur la base de nouveaux modes d'échanges de connaissances pour accroître les "feed-back " entre les usagers finaux et les fournisseurs de la technologie.

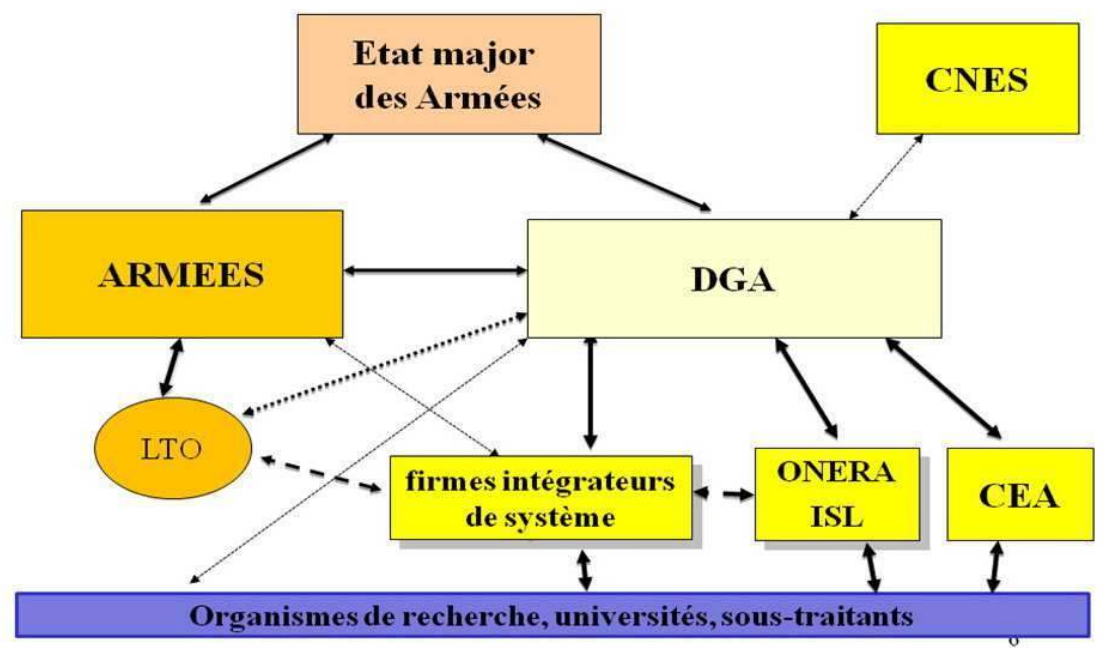

Figure 3 - La situation dela DGA après la réforme de 2003

Malgré ces nouvelles initiatives, l'intensité des échanges reste faible entre acteurs publics et privés. Elle s'est progressivement tarie au cours des années ${ }^{22}$ avec en particulier la réduction des canaux informels de transmission des informations. La formalisation des relations entre la DGA et l'industrie a été concomitante à une formalisation accrue des relations entre la DGA et les Armées, qui s'est opérée à partir de 1997. Les changements initiés en 2003 n'ont pas encore permis de modifier véritablement cette situation. La

les industriels mais elle reste encore limitée à quelques projets pour lesquels des relations de confiance entre les principaux acteurs du domaine ont pu se développer.

22 Ce constat est mentionné et détaillé dans le cadre de la plupart des entretiens réalisés auprès des responsables des firmes, de la DGA, des Armées et des centres de recherche sous tutelle Défense, qui ont participé aux deux réformes (1997-2003).

45 Economie et Institutions $-\mathrm{n}^{\circ} 12 \& 13-2008-2009$ 
création des LTO et l'intensification des liens entre opérationnels et fournisseurs de technologies, constituent, d'une part, une source d'incertitude pour le positionnement de la DGA, et créent, d'autre part, une certaine inertie pour cette dernière. Les ambiguités de son positionnement traduisent donc des tensions sur la nature des changements à la fois technologique et organisationnel. Elles donnent aussi l'opportunité de créer de nouveaux actifs cospécialisés comme nous venons de l'illustrer avec les LTO. Ces changements sont résumés dans le tableau suivant (tableau $\mathrm{n}^{\circ} 1$ ). Tableau $n^{\circ} 1$ :

Les dimensions du changement technologique et institutionnel et leurs incidences sur la division du travail

\begin{tabular}{|c|c|c|c|}
\hline $\begin{array}{l}\text { Période/ } \\
\text { dimension du } \\
\text { changement }\end{array}$ & Avant 1997 & $1997-2003$ & Après 2003 \\
\hline $\begin{array}{l}\text { Dimension } \\
\text { technologique }\end{array}$ & $\begin{array}{l}\text { - Complexité relative } \\
\text { et stabilité } \\
\text { industrielle } \\
\text { - Autonomie des } \\
\text { produits } \\
\text { technologiques les } \\
\text { uns par rapports } \\
\text { aux autres }\end{array}$ & \multicolumn{2}{|c|}{$\begin{array}{l}\text { - Complexité croissante } \\
\text { - Place de l'informatique, l'électronique et } \\
\text { des systèmes de communication et } \\
\text { d'information } \\
\text { - Interdépendances des produits }\end{array}$} \\
\hline $\begin{array}{l}\text { Dimension } \\
\text { institutionnelle }\end{array}$ & $\begin{array}{l}\text { - Contrat Cost + } \\
\text { - Marché administré }\end{array}$ & $\begin{array}{l}\text { - Contrat prix fixe } \\
\text { - Mise en } \\
\text { concurrence }\end{array}$ & $\begin{array}{l}\text { - Risques partagés } \\
\text { - Combinaison } \\
\text { marché/ } \\
\text { collaboration }\end{array}$ \\
\hline $\begin{array}{l}\text { Incidences sur } \\
\text { la spécialisation } \\
\text { des actifs }\end{array}$ & $\begin{array}{l}\text { - Co spécialisation } \\
\text { stable } \\
\text { - Division du travail } \\
\text { relativement corrélée } \\
\text { avec la division des } \\
\text { connaissances }\end{array}$ & \multicolumn{2}{|c|}{$\begin{array}{l}\text { - Co spécialisation accentuée } \\
\text { - Division du travail et des connaissances } \\
\text { évolue rapidement }\end{array}$} \\
\hline
\end{tabular}

\section{LE REPOSITIONNEMENT DE LA DGA}

Le rôle traditionnel du maître d'ouvrage comme propriétaire des grands programmes technologiques et co-concepteur des solutions technologiques (Cohendet \& Lebeau, 1987) a fortement évolué en raison des changements évoqués plus haut. La DGA est amenée à intégrer davantage les firmes dans les processus de production et à exploiter de manière croissante les technologies civiles dans les programmes. Il s'agit, ici, de mieux comprendre les enjeux de cette évolution au niveau de la spécialisation de la DGA, et au niveau de ses compétences et connaissances. 


\subsection{Du "maître d'ouvrage architecte" au " maître d'ouvrage des interfaces ": l'évolution du rôle de la DGA}

Jusqu'au début des années 90, la DGA était située au cœur de la conception des programmes d'armement, et à l'interface entre les firmes et les Armées dont elle traduisait les attentes opérationnelles. Pour assurer la fonction de maître d'ouvrage, elle participait activement à la conception des programmes d'armement. Ainsi, au cours des années 1970, la DGA était capable de réaliser des développements exploratoires même si elle confiait ensuite aux industriels le développement des solutions et leur commercialisation (Mérindol, 2005a). Tant que la DRET ${ }^{23}$ était présente, son rôle d'interlocuteur technique et scientifique fut incontestable.

La position de la DGA était celle d'un " maître d'ouvrage architecte ". Cette institution ne pouvait se limiter à la connaissance architecturale au sens strict et laisser aux fournisseurs et soustraitants l'exclusivité de la connaissance des composants et soussystèmes. Pour coordonner les tâches, elle était amenée à élargir la gamme des champs technologiques dans lesquels elle développait ses compétences. Cette fonction de "maître d'ouvrage architecte " avait également une incidence majeure sur la division des connaissances, car la DGA s'avérait capable de co-spécifier les solutions techniques et d'agir sur ces dernières.

Les mutations technologiques et institutionnelles ont radicalement modifié cette donne. En 1997, la rupture se matérialise par la position de "centrale d'achat " adoptée par la DGA. Le contexte institutionnel des années 1990 a tout d'abord accéléré le processus d'externalisation de la fonction de maitre d'ouvrage pour les programmes technologiques particulièrement complexes. La DGA accorde plus d'autonomie aux firmes et leur offre la possibilité d'acquérir de nouvelles compétences technologiques et organisationnelles, qui sont, en partie, transférées à ces dernières (Guillou et al., 2009). Malgré les priorités données à l'expertise technique, la DGA perd peu à peu sa capacité d'architecte notamment pour les "systèmes de systèmes " (Mérindol, 2005a). L'objectif de réduction de $30 \%$ du personnel24 et des coûts de fonctionnement de cette administration accentue ce mouvement initial. Le poids des industriels dans la conception devient, quant à

23 Direction de la Recherche et des Etudes Techniques de la DGA supprimée en 1997.

24 On estime qu'entre 1988 et 2000 la baisse réelle de l'effectif à été de 30 \%, hors prise en compte du changement de statut de la Direction des constructions navales, arsenal transformé en société publique.

47 Economie et Institutions $-\mathrm{n}^{\circ} 12 \& 13-2008-2009$ 
lui, irréversible et incontournable ${ }^{25}$. Ainsi, la DGA n'est plus à même de suivre l'évolution des connaissances architecturales car, d'une part, les architectures sont devenues plus complexes et, d'autre part, l'intégration des systèmes n'est plus partagée ni maîtrisée dans sa globalité.

La réforme de 2003 va offrir à la DGA la possibilité de rechercher un nouveau positionnement au sein du système national d'innovation. Son intervention porte désormais davantage sur la gestion d'interfaces que sur la co-conception de l'architecture technologique des programmes. La DGA conserve encore une fonction de traduction $\mathrm{du}$ besoin militaire en spécifications techniques; ce rôle peut s'avérer très sophistiqué en raison de la variété croissante des missions dédiées aux Armées et des choix à opérer. Les incidences sur la performance des systèmes d'armes et les coûts des programmes peuvent être majeures. Cette nouvelle position peut être caractérisée de "maître d'ouvrage des interfaces". La DGA doit disposer des connaissances lui permettant d'identifier et de comprendre les diverses compétences des firmes avec lesquelles elle entre en relation, de négocier ainsi que de comprendre la variété des impacts financiers et organisationnels possibles associés aux choix technologiques. En effet, savoir identifier les connaissances et les compétences au sein de chaque métier se révèle essentiel pour définir la liste des sous-systèmes entrant dans la composition du CoPS, même si la capacité de création d'une architecture de programme ne peut plus se faire en interne.

$\mathrm{Au}$ fil des réformes, la DGA a donc délaissé la conception pour se consacrer à la coordination industrielle au sein des systèmes complexes, en recherchant notamment un consensus sur les méthodes de travail des firmes et sous-traitants qui composent cette architecture. Ses activités ont progressivement évolué vers un travail centré sur la définition des spécifications, des règles et modalités de suivi et de contrôle. Ces évolutions sont résumées dans le tableau $\mathrm{n}^{\circ}$ 2.

25 Ce fut le cas du programme SCOAA où, même après la réforme de 2003, la DGA n'est plus intervenue dans la conception technique du programme ni dans l'évaluation de ses performances techniques.

48 Economie et Institutions $-\mathrm{n}^{\circ} 12 \& 13-2008-2009$ 
Tableau $\mathbf{n}^{\circ} 2$ :

Le positionnement de la DGA au rythme des réformes nationales

\begin{tabular}{|c|c|c|c|}
\hline Période & avant 1997 & 1997-2003 & après 2003 \\
\hline $\begin{array}{l}\text { Fonction clé } \\
\text { de la DGA } \\
\text { dans la } \\
\text { division du } \\
\text { travail }\end{array}$ & $\begin{array}{c}\text { Priorité à la } \\
\text { co-conception } \\
\qquad \square \\
\text { Maître d'ouvrage } \\
\text { architecte }\end{array}$ & $\begin{array}{c}\text { Priorité à l'achat } \\
\qquad \square \\
\text { Agence d'achat }\end{array}$ & $\begin{array}{l}\text { Priorité au suivi et } \\
\text { au contrôle } \\
\text { technique } \\
\square \\
\text { Maître d'ouvrage } \\
\text { d'interfaces }\end{array}$ \\
\hline $\begin{array}{l}\text { Position clé } \\
\text { de la DGA } \\
\text { dans le } \\
\text { pilotage de } \\
\text { la R\&D }\end{array}$ & $\begin{array}{c}\text { Logique amont } \\
\qquad \\
\text { Management recherche } \\
\text { et de la technologie }\end{array}$ & $\begin{array}{c}\text { Logique aval } \\
\square \\
\text { Management Produits } \\
\text { Management de la recherche } \\
\text { externalisée }\end{array}$ & $\begin{array}{c}\text { Logique } \\
\text { partenariale } \\
\square \\
\text { Management de la } \\
\text { recherche et } \\
\text { technologie orienté } \\
\text { Réseaux }\end{array}$ \\
\hline
\end{tabular}

L'évolution de la DGA reflète donc de nouveaux types d'interactions et implique de nouvelles modalités pour mobiliser des connaissances et compétences externes. De ce point de vue, la réforme de 1997 amorce une période d'ajustement permettant de passer du modèle de "maître d'ouvrage architecte " à celui de " maître d'ouvrage des interfaces".

\subsection{La gestion du corps des ingénieurs de l'armement et son incidence sur la fonction de maître d'ouvrage}

La construction d'expertise nécessite une accumulation de compétences passées ou anciennement mises en œuvre qu'il s'agit de préserver sur le long terme, voire de renouveler. Les centres techniques et d'essais jouent un rôle clé, à cet égard, car ils permettent de maintenir des compétences tacites minimales pour mieux comprendre les spécifications techniques. Ils offrent aussi l'opportunité de dialoguer avec les industriels et d'entretenir des feedback sur les innovations technologiques mises en œuvre. Pour la DGA, le rôle des centres d'essais dans la construction des compétences reste ambigu car la pratique de la technologie a toujours été limitée. Le tableau suivant retraçant la part du budget de $R \& D$ de Défense consacrée aux travaux exécutés en interne à la DGA (essentiellement des tests et essais), illustre ce processus. 
Tableau 3 : Part des travaux de R\&D réalisés en interne à la DGA (en millions d'euros constants 2004)

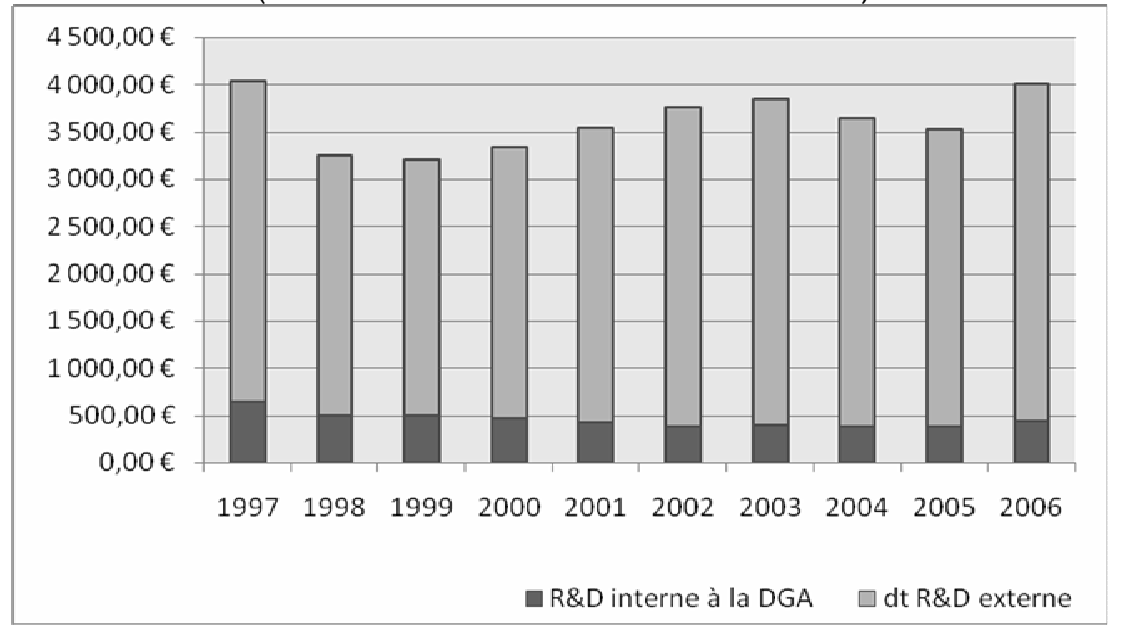

Sources : DGA et Observatoire économique de la Défense et traitement des auteurs

Ce phénomène s'est accentué ces dernières années. Remillieux (2004) met en évidence que les moyens consacrés aux fonctions d'expertise de la DGA sont aujourd'hui principalement associés à la réalisation d'activités de conseil par les centres d'essais, plutôt que directement associés à l'exercice de la technologie et/ou des essais. L'activité principale de certains centres techniques de la DGA confirme cette tendance: plus de $80 \%$ de leurs activités concernent des actions de conseils aux responsables de la gestion des programmes de la DGA à partir de propositions transmises par l'industrie.

Par ailleurs, la construction de l'expertise de la DGA ne peut se comprendre sans faire référence à la place spécifique occupée par le corps des ingénieurs de l'armement, recrutés par le biais de l'Ecole Polytechnique. L'esprit de corps, tel que décrit par Kessler (2005), y est très prononcé. Les ingénieurs de l'armement sont porteurs d'un ensemble de valeurs fondées sur leur histoire, des mythes, une culture technique et un langage commun ainsi qu'une représentation de leur rôle dans l'institution de Défense. Jusqu'au début des années 1990, les ingénieurs de l'armement sont au cœur des réseaux d'innovation et des systèmes de conception des systèmes d'armes. Les relations informelles et la mobilité des ingénieurs entre la Défense et l'industrie y sont denses et obéissent aux règles tacites du fonctionnement du $\operatorname{corps}^{26}$. Adaptée au modèle de relations entre la

26 Les ingénieurs de l'armement étaient aux postes clés des programmes de Défense au CEA, au CNES, à l'ONERA, à la DGA ainsi qu'au sein des principaux industriels impliqués dans la conception des armements.

50 Economie et Institutions $-\mathrm{n}^{\circ} 12 \& 13-2008-2009$ 
Défense et l'industrie pendant la Guerre froide, l'acquisition de l'expertise scientifique et technique des ingénieurs de la DGA, qui se faisait par le biais de nombreux aller-retour entre secteurs public et privés, devient impossible à préserver lors de la réforme 1997. La privatisation et la réorganisation industrielle, tout comme la focalisation de la DGA sur la fonction achat vont aussi réduire les possibilités de construire cette compétence via des allers retours entre l'industrie et la Défense ${ }^{27}$. La réforme de 1997 est donc perçue comme la remise en cause de la position des ingénieurs de l'armement dans la gouvernance des programmes.

Bien que réhabilitant l'importance de la fonction de maître d'ouvrage, la réforme de 2003 peut aussi apparaître comme un recul de ce corps car elle traduit surtout une implication croissante et directe des Armées dans la gestion des programmes. Dans cette évolution, les ingénieurs de l'armement passent progressivement d'un travail centré sur la conception, à un travail centré sur la programmation des études amont. En passant du faire au faire-faire, la perte de compétences issues de la pratique, tend à s'accroître. De plus, les compétences organisationnelles transférées au sein de groupes industriels (Guillou et al., 2009), se trouvent aussi affaiblies. C'est là tout l'enjeu et les limites du nouveau métier de la DGA. Cette dernière est contrainte de focaliser sa capacité technologique non plus sur la maîtrise en profondeur des connaissances nécessaires à la production mais sur la maitrise de leur étendue ${ }^{28}$ afin de savoir où sont localisées les connaissances disponibles dans les firmes et les instituts de recherche. L'étendue des connaissances constitue donc une nouvelle dimension stratégique notamment pour préserver une capacité d'absorption minimale et tenter de retrouver un rôle d'interface à un niveau satisfaisant.

L'influence des ingénieurs de l'armement s'étendait jusque dans la définition des concepts stratégiques dans le domaine militaire et de la politique étrangère. Toutefois, ce modèle fonctionnait en vase clos (Giovachini, 2000). 27 Certains domaines comme les radars ou l'électronique sont sortis très tôt du giron de l'expertise de la DGA. Toutefois, on peut noter quelques exceptions et cas particuliers où les ingénieurs de la DGA partent sur des programmes de recherches amont chez des groupes industriels pour mieux suivre le projet. Mais cette mobilité reste beaucoup moins développée qu'avant la réforme de 1997.

28 L'" étendue " des connaissances fait référence au nombre de champs technologiques dans lesquels la DGA est aujourd'hui active. La "profondeur " renvoie à la maîtrise de deux dimensions majeures :

- les différentes étapes impliquées dans le processus de développement d'un programme d'armes.

- la connaissance liée à la combinaison des composants du programme (connaissance architecturale) et la connaissance concernant chaque composant (connaissance composant).

51 Economie et Institutions $-\mathrm{n}^{\circ} 12 \& 13-2008-2009$ 


\subsection{L'évolution du contenu des compétences de la DGA}

Les compétences nécessaires au "maître d'ouvrage architecte " diffèrent selon le degré de standardisation des soussystèmes, mais une certaine connaissance des caractéristiques fonctionnelles des sous systèmes demeure indispensable. Ces compétences s'appuient aussi sur une capacité de traduction des besoins opérationnels en produits technologiques. La "maîtrise d'ouvrage des interfaces " focalise plus spécifiquement son travail sur le suivi des spécifications techniques. $\mathrm{Si}$ les connaissances architecturales ne sont plus nécessaires, la connaissance des acteurs industriels, des critères de performances des systèmes conçus et la capacité de traduction des besoins militaires, demeurent toujours essentielles. La DGA qui pilote l'intermédiation des relations entre le monde industriel et les Armées, se voit contrainte d'identifier les connaissances critiques et de les faire vivre à travers une mobilisation des réseaux au sein du système national d'innovation. Le passage du métier de "maîtrise d'ouvrage architecte " à celui de "maîtrise d'ouvrage des interfaces" n'est donc pas neutre. Ce changement de posture suppose pour la DGA de donner la priorité à l'acquisition et la pérennisation de certaines compétences technologiques et organisationnelles.

- Premièrement, ses compétences au niveau de l'architecture système tendent à disparaître.

Si les architectes conçoivent les systèmes à partir de soussystèmes, la compétence architecturale ne se réduit pas à savoir combiner des produits ou sous-systèmes pour répondre aux besoins exprimés par les utilisateurs. Le maître d'ouvrage architecte est amené à spécifier non seulement les interfaces que doivent respecter les éléments, mais aussi tout ou partie de leurs caractéristiques fonctionnelles. Il est donc aussi prescripteur de leur contenu. Cette implication de l'architecte dans la conception des éléments peut résulter d'une innovation architecturale (réclamant la mobilisation d'éléments dotés d'un ensemble spécifique de caractéristiques qui n'existe pas sur le marché), ou bien de l'intensité des interdépendances fonctionnelles entre les éléments constitutifs du CoPS.

- Deuxièmement, ses compétences en matière de contrôle de l’intégration évoluent.

Le problème majeur que pose l'intégration d'un système ou produit complexe réside dans l'hétérogénéité de l'ensemble des ressources et compétences à réunir. Cette hétérogénéité est dite

52 Economie et Institutions $-\mathrm{n}^{\circ} 12 \& 13-2008-2009$ 
cognitive car elle ne s'exprime pas seulement au niveau de l'activité productive mais aussi au niveau des connaissances. Il est donc nécessaire de segmenter le travail, de faire dialoguer des spécialistes pour que chacun intègre les contraintes des autres, d'en évaluer le résultat technique, financier, organisationnel. Pour construire une vision partagée des systèmes et faciliter leur intégration, il faut mettre en place des mécanismes particuliers de coordination. Ainsi, en favorisant l'émergence de représentations et de procédures similaires au sein de l'architecture, les organisations comme la DGA peuvent réduire "les coûts de transaction des idées " (Loasby, 2000). Mais l'intérêt du partage ne se limite pas aux représentations, il englobe aussi les outils comme l'illustrent les plateaux de conception communs. Cette organisation des activités n'élimine pas tous les problèmes d'intégration mais peut fortement en réduire les coûts. C'est précisément autour de cette nouvelle forme de contrôle que la DGA peut avoir un rôle dans les performances économiques de l'industrie de la Défense.

- Troisièmement, les compétences pour sélectionner les entreprises se maintiennent même si de nouveaux acteurs, comme les "lead system integrators ", apparaissent à ce niveau.

La sélection des firmes ne peut plus désormais s'effectuer sur la base de la performance de leur offre existante mais à partir de l'évaluation de la qualité des ressources mises au service du programme. D'où la nécessité pour la DGA de disposer d'un niveau minimum de connaissances sur les acteurs des réseaux d'innovation. Cette capacité implique une base étendue de connaissances pour assurer la coordination des différentes firmes aux diverses différentes phases de développement du programme. Lorsque les composants ou sous-systèmes n'existent pas au départ, ce problème de sélection se double du besoin d'inciter les entreprises à entrer dans une logique de co-spécification. Autrement dit, les besoins de coordination ne s'arrêtent pas aux phases de définition et de conception technique des systèmes.

- Enfin, les compétences de la DGA en matière de traduction des besoins militaires deviennent essentielles à sa fonction.

La capacité de traduction du besoin militaire en spécifications techniques est essentielle en raison de la spécificité des métiers et des langages opérationnels. Elle permet de réduire les coûts de coordination entre les usagers finaux et les fournisseurs de technologies. Les enjeux associés à cette compétence sont essentiels. En effet, l'efficacité du recours aux conventions d'assistance à la maîtrise d'ouvrage entre la DGA, l'ONERA et le CEA est aujourd'hui 
limitée par le fait que ces centres de recherche disposent d'une très faible capacité de compréhension du milieu militaire et de traduction du besoin de l'usager.

Au final, jusqu'en 1997, la compétence architecturale devait être maitrisée par le maître d'ouvrage architecte comme par la firme intégrateur de systèmes car ces deux acteurs travaillaient ensemble dans une logique de co-spécification. Le "maître d'ouvrage architecte "devait donc à la fois disposer d'une profondeur et d'une étendue de connaissances lui permettant de co-concevoir le programme et de traduire les enjeux militaires en enjeux technologiques. Cette compétence architecturale servait de base à sa capacité de sélection des entreprises et de contrôle de l'intégration.

A l'inverse, la position de "maître d'ouvrage des interfaces" ne repose pas sur une capacité à définir l'architecture du programme. La compétence architecturale est confiée dans sa globalité aux firmes intégrateurs de systèmes. Toutefois, le maître d'ouvrage des interfaces doit détenir une capacité d'intégration pour sélectionner les firmes, et traduire le besoin militaire. Pour cela, il doit préserver des domaines clés d'évaluation et d'interface et donc préserver une base de connaissances suffisamment étendue. Si l'étendue des connaissances est prioritaire, c'est l'équilibre entre diversité et profondeur des connaissances qui constitue le nouvel enjeu majeur pour la DGA ainsi que pour les acteurs de cette industrie (cf. Prencipe 2000). Le tableau suivant synthétise ces mutations.

Tableau 4 : Les connaissances et les compétences de la DGA et leur évolution

\begin{tabular}{|l|c|c|}
\hline & $\begin{array}{c}\text { avant 1997 } \\
\text { Maître d'ouvrage architecte }\end{array}$ & $\begin{array}{c}\text { après 2003 } \\
\text { Maître d'ouvrage des interfaces }\end{array}$ \\
\hline $\begin{array}{l}\text { Compés } \\
\text { Dimension centrale }\end{array}$ & $\begin{array}{c}\text { Compétences architecturales } \\
\text { Compétences de contrôle sur } \\
\text { l'intégration } \\
\text { Compétences de sélection } \\
\text { Compétences de traduction des } \\
\text { besoins militaires }\end{array}$ & $\begin{array}{c}\text { Compétences de contrôle sur la } \\
\text { performance } \\
\text { Compétences de sélection } \\
\text { Compétences de traduction des } \\
\text { besoins militaires }\end{array}$ \\
\hline Connaissances & $\begin{array}{c}\text { Technologique } \\
\text { Etendue et profondeur des } \\
\text { connaissances }\end{array}$ & Priorité à l'étendue des connaissances \\
\hline
\end{tabular}

54 Economie et Institutions $-\mathrm{n}^{\circ} 12 \& 13-2008-2009$ 


\section{Conclusion}

Les mutations technologiques et institutionnelles ont eu un impact majeur sur l'industrie de la Défense. En effet, la nouvelle architecture industrielle a généré, d'une part, de nouvelles modalités d'innovation entre les partenaires publics et privés et, d'autre part, de nouveaux types d'interactions entre les acteurs publics. Dans ce modèle d'innovation plus ouvert, selon la terminologie de Chesbrough (2003), le rôle et les compétences de chaque acteur ont été modifiés. Suite au renforcement de leur rôle dans l'intégration des systèmes, les firmes ont acquis une position centrale au sein de l'architecture et renforcé leur position dans les projets de recherche amont financés par l'Etat. Ces dernières ont également bénéficié d'un transfert de compétences nouvelles et d'une d'autonomie grandissante lors de la mise en place des politiques de recherche et d'innovation. Pour tenter de rester un acteur non négligeable au sein du système national d'innovation, et surtout pour conserver un degré minimal d'expertise, la DGA a eu recours à la mise en place de nombreux actifs co-spécialisés (les LTO en sont une bonne illustration). Dans cette nouvelle architecture, le poids du " maître d'ouvrage " traditionnel s'est amoindri au profit de celui des " maîtres d'œuvre ", c'est-à-dire des firmes qui avaient la capacité technologique d'offrir des innovations répondant aux caractéristiques technologiques et organisationnelles des CoPs. L'évolution " maître d'ouvrage architecte " / " maître d'ouvrage des interfaces " a également diminué le poids de la DGA au sein du SNI au profit d'autres institutions publiques. Pour maintenir une "capacité d'absorption minimale " et pour suivre l'ensemble des nouveaux métiers technologiques que désormais elle ne pratique plus, la DGA a donc multiplié les interactions avec les acteurs publics et privés. C'est là tout l'enjeu du re-positionnement de cette institution, qui pour ne pas voir son rôle restreint à celui d'un simple acheteur et négociateur de contrat industriels, doit au contraire maintenir un haut niveau de compétences et d'expertise technique par le biais de nouvelles formes de partenariats sur le long terme. Enfin d'une manière plus générale, les nouvelles formes de partenariat et de cospécialisation sont nécessaires pour assurer le suivi, la pérennité et la maintenance des projets complexes. L'exemple de la maintenance dans la construction navale montre pourquoi dans de nombreux domaines, l'expertise technologique publique est toujours critique, même si elle peut être remise en cause. 
Annexe 1: Liste des acronymes

$\begin{array}{ll}\text { CEA } & \text { Commissariat à l'Energie Atomique } \\ \text { CGARm } & \text { Conseil Général de l'Armement. } \\ \text { CNES } & \text { Centre National d'Etudes Spatiales } \\ \text { CNRS } & \text { Centre National de Recherches Scientifiques } \\ \text { CoPS } & \text { Complex products and systems } \\ \text { DAM } & \text { Direction des Applications Militaires (au sein du CEA) } \\ \text { DGA } & \text { Délégation Générale pour l'Armement } \\ \text { DRET } & \text { Direction de la Recherche et des Etudes Techniques } \\ \text { EADS } & \text { European Aeronautic Defence and Space Company } \\ \text { INRIA } & \text { Institut National de Recherche en Informatique et en } \\ & \text { Automatique } \\ \text { LSI } & \text { Lead System Integrator } \\ \text { LTO } & \text { Laboratoires Technico-Opérationnels } \\ \text { MoD: } & \text { Ministry of Defence (Royaume Uni) } \\ \text { ONERA } & \text { Office National d'Etudes et de Recherches } \\ & \text { Aérospatiales } \\ \text { RRIT } & \text { Réseaux de Recherche et d'Innovation technologiques } \\ \text { SNI } & \text { Système National d'Innovation } \\ \text { SCCOA } & \text { Système de Communication et de Commandement des } \\ & \text { Opérations Aériennes. }\end{array}$




\section{BIBLIOGRAPHIE}

ACHA V., BRUSONI S. (2008) "The changing Governance of Knowledge in Avionics", pp.43-57.

Economics of Innovation and New Technology, 17, (102),

AVADYKIAN A., COHENDET P., DUPOUET O. (2005), "A study of Military Innovation Diffused Based on two Case Studies", in LLERENA P. \& MATT M. (ed by), Innovation Policy in a Knowledge Based Economy, Germany : Springer pp. 161-189.

BAILEY GRASSO V. (2007), "Defense acquisition: use of lead system integrators (LSIs) - Background, Oversight Issues and option for Congress", CRS Report for Congress, the library of Congress, Washington, 26 mars.

BALDWIN C., CLARK K. (2000) Design Rules: The Power of Modularity, Volume 1, The MIT Press, Cambridge, Massachusetts.

BELLEVAL C. (2006), " De la remise en question des pratiques des grands projets de haute technologie d'Etat à celle des missions du maître d'ouvrage : le cas d'un programme spatial ", n spécial en l'honneur de Fernand Amesse, Management international $\mathrm{n}^{\circ} 10$, pp. 55-64.

BERNARD J.L \& CARRE A. (2005), Les conditions d'exécution des grands programmes de Défense, Rapport d'information $\mathrm{n}^{\circ} 1922$,

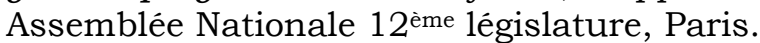

BRUSONI S., PRENCIPE A. (2006) “ Making Design Rules: A Multidomain Perspective” Organization Science, 17, pp.179-189.

BRUSONI S., PRENCIPE A. \& PAVITT K. (2001), "Knowledge specialisation, organizational coupling, and the boundaries of the firm : why do firms know more than they make?", Administrative science quarterly, 46 (4), pp. 597-621.

COHEN W. \& LEVINTHAL D. A. (1990), "Absorptive capacity : a new perspective on learning and innovation", Administrative Science Quartely, 35 (1), pp.128-152.

CHESBROUGH H. (2003), Open Innovation: the new imperative for creating and profiting technology, Boston : Harward Business School press.

CHESNAIS F. \& SERFATI C. (1992), L'armement en France : genèse, ampleur et coût d'une industrie, coll. Economie et sciences sociales, Paris : Nathan.

COHENDET P. \& LEBEAU A (1987), Choix stratégiques et grands programmes civils, Paris : CPE- Economica, 189 pages.

DAVIES A. \& BRADY T. (2000) "Organisational capabilities and learning in complex product systems: towards repeatable solutions", Research Policy, 29 (7-8), pp.931-953. 
DAVIES A. \& HOBDAY M. (2005) The business of projects: managing innovation in complex products and systems, Cambridge : Cambridge University Press.

DELEGATION GENERALE POUR L'ARMEMENT (1997), "La réforme de la DGA ", revue l'Armement, n'spécial, ministère de la Défense, décembre 1996-janvier 1997.

DELEGATION GENERALE POUR L'ARMEMENT (2004), Pour une autonomie compétitive en Europe: la politique d'acquisition $d u$ ministère de la Défense, Ministère de la Défense, http://www.defense.gouv.fr/dga/layout/set/popup/content/vie $\mathrm{w} /$ full/64595, consulté le 9 mars 2003

DELEGATION GENERALE POUR L'ARMEMENT (2006), Politique et Objectifs Scientifiques, Ministère de la Défense, Paris

DEPEYRE C. \& DUMEZ H. (2007), "Le rôle du client dans les stratégies de coopétition ", Revue française de gestion, 7 (176), pp.99-110.

DIMAGGIO P.J. \& POWELL W.W. (1983), "The Iron Cage Revisited: Institutional Isomorphism and Collective Rationality in Organizational Fields", American Sociological Review, 48, pp. 147160.

DOWDALL P., (2004), "Chains, Networks and Shifting paradigms:

The UK defence Industry Supply System", Defence and Peace

Economics, 15 (6), pp.535-550.

FLOOD S. \& RICHARD P. (2006), "An assessment of the lead systems integrator concept as applied to the future combat system program", Defense Acquisition review journal, December 2005march 2006, pp. 357-373.

FROMION Y. (2005), Rapport d'information sur la recherché de Défense et de Sécurité, Assemblée nationale, rapport $n^{\circ} 2150$, Paris.

GIOVACHINI L. (2000), L'armement français au XXème siècle : une politique à l'épreuve de l'histoire, Les cahiers de l'armement, Paris : Ellipses.

GUICHARD R. (2005), "Suggested repositionning of Defence R\&D within the French System of Innovation", Technovation, 25(3), pp. 195-201

GUILLOU S., LAZARIC N., LONGHI C., ROCHHIA S. (2009) "The French defence industry in the knowledge management era: A historical overview and evidence from empirical data", Research Policy, 38, issue 1, 170-180

HARTLEY K. (1995), "Industrial Policies in the Defense Sector", chapter 16 in Handbook of Defense Economics vol.1, edited by Hartley K. \& SANDLER T., Elsevier North Holland, pp.459-489.

HOBDAY M. (1998), "Product complexity, innovation and industrial organisation", Research Policy, 26, pp. 689-710 
HOBDAY M. (2000), "The project-based organization: an ideal form for managing complex products and systems?", Research Policy, 29 (7-8), pp.871-893.

HOBDAY M., DAVIES A, PRENCIPE A. (2005), "Systems integration: a core capability of the modern corporation", Industrial and Corporate Change, 14 (6), pp.1109-1143.

JACOBIDES M.G. (2006) "The architecture and design of organizational capabilities", Industrial and Corporate Change, 15 (1), pp.151-171.

JACOBIDES M.G., KNUSDEN T., AUGIER M. (2006), "Benefiting from Innovation: Value creation, value appropriation and the role of industry architecture", Research Policy 35, pp. 1200-1221

JACOBIDES M.G. (2007) "Pour une approche stratégique des architectures sectorielles ", Le Libellio, 3 (2), p. 28-34.

KAUSAL T., HUMILY G., TAYLOR T. \& ROLLER P. (1999), A comparison of the Defense Acquisition systems of France, Great Britain, Germany and United States, Defense Systems management college, Virginia, 22060 5565, septembre

KESSLER .C (2005), L'esprit de corps dans les grands corps de l'Etat en France, Working paper du CERSA, Université Paris II Panthéon Assas.

KIRAT T., BAYON D., BLANC H. (2003), Maîtriser les coûts des programmes d'armement: une analyse comparative de la réglementation des marchés industriels d'armement en France, au Royaume-Uni et aux Etats-Unis, Les rapports de l'Observatoire économique de la Défense, Paris : La Documentation française.

LAREDO P. \& MUSTAR P. (2001), "French Research and Innovation Policy: Two Decades of Transformation", in LAREDO P. \& MUSTAR P. (ed.by), Research and Innovation Policies in the Global Economy : An international Comparative Analysis, Cheltenham : Edward Elgar, pp. 447-496.

LINIERE CASSOU M. (2000), Les études amont des programmes d'armement dans le domaine de la Défense et de l'aéronautique, rapport d'information $\mathrm{n}^{\circ} 2793$, Commission de la Défense nationale, Assemblée Nationale.

MATTHEWS D. \& COLLIER P. (2000), "Assessing the value of a C4ISREW System-of-Systems Capability", working paper, Australia: Joint systems Branch DST.

MERINDOL V. (2005a), "La Défense dans les réseaux d'innovation : une analyse en termes de compétences" Revue d'Economie Industrielle, décembre, pp. 45-64

MERINDOL V. (2005b), "Defense R\&DTE and knowledge management: A new inquiry into public-private coordination", Defense and Security Analysis, 21 (2), june, pp.159-177. 
O'SULLIVAN A. (2006) "Why tense, unstable, and diverse relations are inherent in co-designing with suppliers: an aerospace case study", Industrial and Corporate Change, 15(2), pp.221-250.

PAVITT K. [2003], "Specialization and System Integration: Where Manufacture and

PESTRE D. (2001), " La création de la DMA en 1961 ", Revue Scientifique et Technique de la Défense 2001-4, Délégation Générale de l'Armement, pp.71-76.

PRENCIPE A. (1997) "Technological Capabilities and Product Evolutionary Dynamics: a case study from the aero engine industry". Research Policy, 25, pp.1261-1276.

PRENCIPE A. (2000), "Breath and depth of technological capabilities in COPS: the case of aircraft engine control system" Research Policy, 29, pp.895-911.

REMILLIEUX Y. (2004), "La contribution de la DCE aux études amont de la DGA. ", revue l'Armement $\mathrm{n}^{\circ} 85$, numéro spécial sur les études amont: les démonstrateurs, Délégation générale pour l'armement, mars, pp.25-33

SEO M.G. CREED D. (2002), "Institutional Contradictions, Praxis, and Institutional Change: a Dialectical Perspective", Academy of Management Review, 27 (2), pp. 222-247.

SERFATI C. (2001) "The Adaptability of the French Armament Industry in an Era of Globalization", Industry and Innovation, 8. $\mathrm{n}^{\circ} 2$.

SERFATI C. (2008) "Financial dimensions of transnational corporations, global value chain and technological innovation", Journal of Innovation Economics, 2 (2), pp.35-61

TEECE D.J (1986) "Profiting from technological innovation: Implications for integration, collaboration, licensing and public policy", Research Policy , 15 (6), pp.285-305

VERSAILLES D.W. (2005), "Le maître d'oeuvre dans les programmes d'armement : de l'émergence à la consolidation des réseaux de connaissances ", Revue d'Economie Industrielle, décembre, pp. 83-105. 